\title{
ON ANALYTIC SETS IN TOPOLOGICAL SPACES
}

\author{
BY \\ MAURICE SION
}

1. Introduction. With a given family $H$ of subsets of a space $X$ there are associated two classical families: Borel $H$, the smallest family containing $H$ and closed under countable unions and complementation, and Souslin $H$, the family obtained by applying the operation $A$ defined by Souslin (see Definition 2.11). The case where $X$ is a complete separable metric space and $H$ is the family of all closed sets in $X$ has been extensively studied and various relations between the Borel sets, Souslin sets, and continuous images of $G_{\delta}$ sets established (see $[4 ; 5 ; 7]$ ).

The case where $X$ is a Hausdorff space and $H$ is the family of all compact sets in $X$ has been considered by Choquet $[1 ; 2 ; 3]$ and Šneider $[8 ; 9 ; 10]$ who replaced Borel $H$ by Borelian $H$, the smallest family containing $H$ and closed under countable unions and intersections. However, the extensions of some of the major results in the classical theory were left as open questions (see [2]). In this paper, we extend some of these classical results. In particular, we prove the following:

Two disjoint Souslin sets can be separated by two disjoint Borelian sets (Theorem 4.3). If $X$ is the countable union of compact sets, then the Souslin sets coincide with the continuous images of $K_{\sigma \delta}$ sets, where $K$ is the family of all compact sets in some Hausdorff space $X^{\prime}$ (Theorem 5.5). Taking $K$ and $X$ as above, if the difference between two sets in $K$ is a $K_{\sigma}$ then the continuous, countable-to-one image of a $K_{\sigma \delta}$ is Borelian (Theorem 6.10).

After this paper was presented for publication, the author learned that Theorems 5.4 and 5.5 had also been proved by G. Choquet in [3]. These results were obtained independently of each other and the methods of proof are somewhat different.

2. Notation and basic definitions. We follow Choquet [2] in using the terminology Borelian $H$, Souslin $H$, and analytic in $X$, but extend somewhat the definition of the latter.

2.1. A non-negative integer $n$ contains all smaller non-negative integers, i.e., $m \in n$ iff $m<n$ and $m$ is a non-negative integer. Thus, 0 is both the empty set and the smallest non-negative integer.

2.2. $\omega$ denotes the set of all non-negative integers.

2.3. $S_{n}$ denotes the set of all $n$-tuples of non-negative integers, i.e., functions on $n$ to $\omega$.

Presented to the Society, November 21, 1959; received by the editors September 28, 1959. 
2.4. $S^{\prime}$ denotes the set of all sequences of non-negative integers, i.e., functions on $\omega$ to $\omega$.

2.5. $f / A$ denotes the restriction of $f$ to $A$.

2.6. $\mathcal{F}(X)$ denotes the family of all closed sets in $X$.

2.7. $K(X)$ denotes the family of all compact sets in $X$.

2.8. $A$ is a $K_{\sigma}(X)$ iff there exists a sequence $B$ such that $B_{n} \in K(X)$ for every $n \in \omega$ and $A=\bigcup_{n \in \omega} B_{n}$.

2.9. $A$ is a $K_{\sigma \delta}(X)$ iff there exists a sequence $B$ such that $B_{n}$ is a $K_{\sigma}(X)$ for every $n \in \omega$ and $A=\bigcap_{n \in \omega} B_{n}$.

2.10. $A$ is Borelian $H$ iff $A$ belongs to the smallest family $B$ such that: $H \subset B$ and if $C_{n} \in B$ for every $n \in \omega$ then $\bigcup_{n \in \omega} C_{n} \in B$ and $\cap_{n \in \omega} C_{n} \in B$.

2.11. $A$ is Souslin $H$ iff there exists a function $E$ on $\bigcup_{n \in \omega} S_{n}$ to $H$ such that

$$
A=\bigcup_{s \in S^{\prime}} \bigcap_{n \in \omega} E(s / n) .
$$

2.12. $A$ is analytic in $X$ iff, for some Hausdorff space $X^{\prime}, A$ is the continuous image of a $K_{\sigma \delta}\left(X^{\prime}\right)$.

2.13. $\pi$ denotes the projection onto the first coordinate space, i.e., $\pi(x, y)=x$.

3. Preliminary lemmas. Throughout this section we assume that $X$ and $X^{\prime}$ are topological spaces.

3.1. Lemma. If, for every $n \in \omega, A_{n} \subset X \times X^{\prime}$ and, for every $x \in X$, $\left(A_{n} \cap \pi^{-1}\{x\}\right)$ is compact in the product topology and the family

$$
\bigcup_{n \in \omega}\left\{\left(A_{n} \cap \pi^{-1}\{x\}\right)\right\}
$$

forms a nest then

$$
\pi\left(\bigcap_{n \in \omega} A_{n}\right)=\bigcap_{n \in \omega} \pi\left(A_{n}\right)
$$

Proof. Clearly

$$
\pi\left(\bigcap_{n \in \omega} A_{n}\right) \subset \bigcap_{n \in \omega} \pi\left(A_{n}\right) .
$$

Suppose $x \in \bigcap_{n \in \omega} \pi\left(A_{n}\right)$. Then, for every $n \in \omega, A_{n} \cap \pi^{-1}\{x\} \neq 0$ and, since a nest of nonvoid compact sets has a nonvoid intersection, we conclude $\bigcap_{n \in \omega} A_{n} \cap \pi^{-1}\{x\}=\bigcap_{n \in \omega}\left(A_{n} \cap \pi^{-1}\{x\}\right) \neq 0$ i.e.,

$$
x \in \pi\left(\bigcap_{n \in \omega} A_{n}\right) \text {. }
$$

3.2. Lemma. Suppose for every $n \in \omega, p \in S_{n}, q \in S_{n}, p \neq q$, we have $A(p)$ $\cap A(q)=0$ and $A(p) \subset A(p /(n-1))$ for $n>0$. Then 


$$
\bigcap_{n \in \omega} \bigcup_{p \in S_{n}} A(p)=\bigcup_{s \in S^{\prime}} \bigcap_{n \in \omega} A(s / n) .
$$

Proof. Clearly

$$
\bigcup_{\cdot \in S^{\prime}} \bigcap_{n \in \omega} A(s / n) \subset \bigcap_{n \in \omega} \bigcup_{p \in S_{n}} A(p) .
$$

Suppose $x \in \bigcap_{n \in \omega} \mathrm{U}_{p \in S_{n}} A(p)$. Then for every $n \in \omega$ there exists a unique $p(n) \in S_{n}$ such that $x \in A(p(n))$. Since $A(p(n+1)) \subset A(p(n+1) / n)$, we must have $p(n+1) / n=p(n)$. Thus, there exists $s \in S^{\prime}$ such that $s / n=p(n)$ for $n \in \omega$ and $x \in \bigcap_{n \in \omega} A(s / n)$.

3.3. Lemma. Suppose $X$ is Hausdorff, $D \subset X^{\prime}, f$ is continuous on $D$ to $X$, $E=\{(x, y): y \in D$ and $x=f(y)\}$, and $\bar{E}$ is the closure of $E$ in the product topology. Then

$$
\bar{E} \cap(X \times D)=E .
$$

Proof. Clearly $E \subset \bar{E} \cap(X \times D)$. If $(x, y) \in \bar{E} \cap(X \times D)$, then $y \in D$ and $(x, y)$ is the limit of points $\left(x^{\prime}, y^{\prime}\right)$, where $\left(x^{\prime}, y^{\prime}\right) \in E$, i.e., $y^{\prime} \in D$ and $x^{\prime}=f\left(y^{\prime}\right)$. Then the $y^{\prime}$ tend to $y$ and the $x^{\prime}$ tend to $x$ and, by continuity, also to $f(y)$. Since $X$ is Hausdorff, we have $x=f(y)$ and hence $(x, y) \in E$.

3.4. Lemma. If $X$ is Hausdorff, $D$ is a $K_{\sigma \delta}\left(X^{\prime}\right)$ and $f$ is continuous on $D$ to $X$ and $A=f(D)$ then there exist $E$ and $C(n, k)$, for $n \in \omega$ and $k \in \omega$, such that:

(i) $E=\bigcap_{n \in \omega} \cup_{k \in \omega} C(n, k) \subset X \times X^{\prime}$.

(ii) $A=\pi(E)$.

(iii) $C(n, k) \cap \pi^{-1}\{x\}$ is compact in the product topology for every $x \in X$.

(iv) $\pi\left(\bigcap_{j \in n} C\left(j, s_{j}\right)\right) \in \mathcal{F}(X)$ for every $s \in S^{\prime}$ and $n \in \omega$.

Proof. Let

$$
D=\bigcap_{n \in \omega} \bigcup_{k \in \omega} d(n, k),
$$

where $d(n, k) \in K\left(X^{\prime}\right)$ for every $n \in \omega$ and $k \in \omega$, and

$$
E=\{(x, y): y \in D \text { and } x=f(y)\} .
$$

Thus, $A=\pi(E)$. Let $\bar{E}$ be the closure of $E$ in the product topology and

$$
C(n, k)=\bar{E} \cap(X \times d(n, k)) \text {. }
$$

Then by 3.3 ,

$$
E=\bar{E} \cap(X \times D)=\bigcap_{n \in \omega} \bigcup_{k \in \omega} C(n, k)
$$

Since

$$
C(n, k) \cap \pi^{-1}\{x\}=\bar{E} \cap(\{x\} \times d(n, k))
$$

we see that (iii) is checked. 
Finally, let $s \in S^{\prime}$ and $n \in \omega$. If $x \in X-\pi\left(\bigcap_{j \in n} C\left(j, s_{j}\right)\right)$ then $\{x\} \times d\left(0, s_{0}\right)$ is compact and has no point in common with $\bigcap_{j \in n} C\left(j, s_{j}\right)$ which is closed in the product topology. Hence there exists a neighborhood $U$ of $x$ such that $U \times d\left(0, s_{0}\right)$ has no points in common with $\bigcap_{j \in n} C\left(j, s_{j}\right)$ and therefore $U \cap \pi\left(\bigcap_{j \in n} C\left(j, s_{j}\right)\right)=0$. Thus, $\pi\left(\bigcap_{j \in n} C\left(j, s_{j}\right)\right)$ is closed in $X$.

4. Properties of Souslin sets.

4.1. Theorem. If, for every $n \in \omega, A_{n}$ is Souslin $H$ then

$$
\bigcup_{n \in \omega} A_{n} \text { and } \bigcap_{n \in \omega} A_{n} \text { are Souslin } H \text {. }
$$

Proof. See [4].

4.2. Theorem. If $\mu$ is a Caratheodory outer measure and all the sets in $H$ are $\mu$-measurable then the Souslin $H$ sets are $\mu$-measurable.

Proof. See [6].

4.3. Theorem. If $X$ is Hausdorff, $A$ and $B$ are Souslin $K(X)$, and $A \cap B$ $=0$ then there exist $A^{\prime}$ and $B^{\prime}$ such that: $A^{\prime}$ and $B^{\prime}$ are Borelian $K(X), A \subset A^{\prime}$, $B \subset B^{\prime}$, and $A^{\prime} \cap B^{\prime}=0$.

Proof. Let

$$
\begin{aligned}
& A=\bigcup_{s \in S^{\prime}} \bigcap_{n \in \omega} E(s / n), \\
& B=\bigcup_{s \in S^{\prime}} \bigcap_{n \in \omega} F(s / n),
\end{aligned}
$$

where, for $s \in S^{\prime}$ and $n \in \omega, E(s / n)$ and $F(s / n)$ are compact in $X$. We also assume, without loss of generality, that

$$
E(s /(n+1)) \subset E(s / n) \text { and } F(s /(n+1)) \subset F(s / n) .
$$

For every $n \in \omega$ and $p \in S_{n}$, let

$$
\begin{aligned}
\mathcal{S}(p) & =\left\{p^{\prime}: p^{\prime} \in S_{n+1} \text { and } p^{\prime} / n=p\right\}, \\
\mathcal{S}^{\prime}(p) & =\left\{s: s \in S^{\prime} \text { and } s / n=p\right\}, \\
E^{\prime}(p) & =\bigcup_{s \in \mathcal{S}^{\prime}(p)} \bigcap_{m \in \omega} E(s / m), \\
F^{\prime}(p) & =\bigcup_{s \in \mathcal{S}^{\prime}(p)} \bigcap_{m \in \omega} F(s / m) .
\end{aligned}
$$

We first check the following:

Statement. If $n \in \omega, p \in S_{n}, q \in S_{n}, E^{\prime}(p)$ and $F^{\prime}(p)$ cannot be separated by Borelian $K(X)$ sets, then for some $p^{\prime} \in \mathcal{S}(p)$ and $q^{\prime} \in \mathcal{S}(q), E^{\prime}\left(p^{\prime}\right)$ and $F^{\prime}\left(q^{\prime}\right)$ cannot be separated by Borelian $K(X)$ sets. 
If the statement is false, then for every $p^{\prime} \in \mathcal{S}(p)$ and $q^{\prime} \in \mathcal{S}(q)$ there exist Borelian $K(X)$ sets $\alpha\left(p^{\prime}, q^{\prime}\right)$ and $\beta\left(p^{\prime}, q^{\prime}\right)$ such that

$$
E^{\prime}\left(p^{\prime}\right) \subset \alpha\left(p^{\prime}, q^{\prime}\right), \quad F^{\prime}\left(q^{\prime}\right) \subset \beta\left(p^{\prime}, q^{\prime}\right), \quad \alpha\left(p^{\prime}, q^{\prime}\right) \cap \beta\left(p^{\prime}, q^{\prime}\right)=0 .
$$

Let

$$
\begin{aligned}
& \alpha^{\prime}=\bigcup_{p^{\prime} \in \mathcal{S}(p)} \bigcap_{q^{\prime} \in \mathcal{S}(q)} \alpha\left(p^{\prime}, q^{\prime}\right), \\
& \beta^{\prime}=\bigcup_{q^{\prime} \in \mathcal{S}(q)} \bigcap_{p^{\prime} \in \mathcal{S}(p)} \beta\left(p^{\prime}, q^{\prime}\right) .
\end{aligned}
$$

Then, since $S(p)$ and $\delta(q)$ are countable, we see that $\alpha^{\prime}$ and $\beta^{\prime}$ are Borelian $K(X), \alpha^{\prime} \cap \beta^{\prime}=0$,

$$
\begin{aligned}
& E^{\prime}(p)=\bigcup_{p^{\prime} \in \mathcal{S}(p)} E^{\prime}\left(p^{\prime}\right) \subset \alpha^{\prime}, \\
& F^{\prime}(q)=\bigcup_{q^{\prime} \in \mathcal{S}(q)} F^{\prime}\left(q^{\prime}\right) \subset \beta^{\prime},
\end{aligned}
$$

in contradiction to the hypothesis of the statement.

Returning to the proof of the theorem, if $A$ and $B$ cannot be separated by Borelian $K(X)$ sets then there exist $s \in S^{\prime}$ and $t \in S^{\prime}$ such that, for every $n \in \omega, E^{\prime}(s / n)$ and $F^{\prime}(t / n)$ cannot be separated by Borelian $K(X)$ sets. Let

$$
E^{\prime \prime}=\bigcap_{n \in \omega} E(s / n) \text { and } F^{\prime \prime}=\bigcap_{p \in \omega} F(t / n) .
$$

Then $E^{\prime \prime}$ and $F^{\prime \prime}$ are compact in $X, E^{\prime \prime} \subset A, F^{\prime \prime} \subset B$ and hence $E^{\prime \prime} \cap F^{\prime \prime}=0$. Since $X$ is Hausdorff, there exist open sets $U$ and $V$ such that $E^{\prime \prime} \subset U$, $F^{\prime \prime} \subset V, U \cap V=0$. Then, for some $n \in \omega, E(s / n) \subset U$ and $F(t / n) \subset V$ so that $E^{\prime}(s / n)$ and $F^{\prime}(t / n)$ can be separated by compact sets, $E(s / n)$ and $F(t / n)$, in contradiction to the above.

5. Relation between analytic and Souslin sets. The main theorem in this section is 5.1. Theorem 5.3 was stated by Choquet [2], but we give a proof here for the sake of completeness.

5.1. Theorem. If $X$ is Hausdorff and $A$ is analytic in $X$ then $A$ is Souslin $\mathfrak{F}(X)$.

Proof. In view of Lemma 3.4, let $A=\pi(E)$ where

$$
E=\bigcap_{n \in \omega} \bigcup_{k \in \omega} C(n, k) \subset X \times X^{\prime},
$$

for some topological space $X^{\prime}$, and, for every $x \in X, n \in \omega, k \in \omega, s \in S^{\prime}$, $C(n, k) \cap \pi^{-1}\{x\}$ is compact in the product topology and $\pi\left(\bigcap_{j \in n} C\left(j, s_{j}\right)\right)$ is closed in $X$. For every $s \in S_{n}$, let

$$
A^{\prime}(s)=\pi\left(\bigcap_{j \in n} C\left(j, s_{j}\right)\right)
$$


We shall show that

$$
A=\bigcup_{s \in S^{\prime}} \bigcap_{n \in \omega} A^{\prime}(s / n) .
$$

(i) Suppose $x \in A$. Then, for some $y,(x, y) \in E$ i.e., there exists $s \in S^{\prime}$ such that, for every $n \in \omega$,

$$
(x, y) \in C\left(n, s_{n}\right)
$$

Hence

$$
(x, y) \in \bigcap_{j \in n} C\left(j, s_{j}\right)
$$

i.e.

$$
x \in \pi\left(\bigcap_{j \in n} C\left(j, s_{j}\right)\right)=A^{\prime}(s / n)
$$

and

$$
x \in \bigcap_{n \in \omega} A^{\prime}(s / n) .
$$

(ii) Suppose, for some $s \in S^{\prime}$,

$$
x \in \bigcap_{n \in \omega} A^{\prime}(s / n) .
$$

Then, by Lemma 3.1,

$$
\begin{gathered}
x \in \bigcap_{n \in \omega} \pi\left(\bigcap_{j \in n} C\left(j, s_{j}\right)\right)=\pi\left(\bigcap_{n \in \omega} \bigcap_{j \in n} C\left(j, s_{j}\right)\right)=\pi\left(\bigcap_{j \in \omega} C\left(j, s_{j}\right)\right) \\
\subset \pi(E)=A .
\end{gathered}
$$

5.2. Corollary. If $X$ is Hausdorff, $B$ is a $K_{\sigma}(X), A \subset B$, and $A$ is analytic in $X$ then $A$ is Souslin $K(X)$.

5.3. Corollary. If $X$ is Hausdorff and $A$ is analytic in $X$ then $A$ is $\mu$-measurable for all measures $\mu$ on $X$ such that closed sets are $\mu$-measurable. in $X$.

5.4. Theorem. If $X$ is Hausdorff and $A$ is Souslin $K(X)$ then $A$ is analytic

Proof. We order the elements of $S^{\prime}$ as follows:

If $s \in S^{\prime}, t \in S^{\prime}, s \neq t$, let $n$ be the first integer such that $s_{n} \not t_{n}$ and then set $s<t$ iff $s_{n}<t_{n}$. For $p \in S_{n}$, let

$$
s(p)=\left\{s: s \in S^{\prime} \text { and } s / n=p\right\} .
$$

Then, in the topology on $S^{\prime}$ induced by the above ordering, $s(p)$ is a $K_{\sigma}\left(S^{\prime}\right)$. If $A$ is Souslin $K(X)$, let 


$$
A=\bigcup_{s \in S^{\prime}} \bigcap_{n \in \omega} E(s / n)
$$

where, for every $s \in S^{\prime}$ and $n \in \omega, E(s /(n+1)) \subset E(s / n) \in K(X)$. Let $X^{\prime}$ $=X \times S^{\prime}$, with the product topology,

$$
\begin{aligned}
B_{n} & =\bigcup_{p \in S_{n}}(E(p) \times \delta(p)), \\
D & =\bigcap_{n \in \omega} B_{n},
\end{aligned}
$$

then $D$ is a $K_{\sigma \delta}\left(X^{\prime}\right)$, since $S_{n}$ is countable. We shall show that $A=\pi(D)$.

(i) If $x \in A$ then for some $s \in S^{\prime}$ :

$$
x \in \bigcap_{n \in \omega} E(s / n)
$$

and

$$
(x, s) \in \bigcap_{n \in \omega}(E(s / n) \times s(s / n)) \subset D .
$$

(ii) If $x \in \pi(D)$, then by Lemma 3.2

$$
\begin{aligned}
x \in \pi\left(\bigcap_{n \in \omega} \bigcup_{p \in S_{n}}(E(p) \times s(p))\right) & =\pi\left(\bigcup_{s \in S^{\prime}} \bigcap_{n \in \omega}(E(s / n) \times s(s / n))\right) \\
& \subset \bigcup_{s \in S^{\prime}} \bigcap_{n \in \omega} E(s / n)=A .
\end{aligned}
$$

5.5. Corollary. If $X$ is Hausdorff, $A \subset B$, and $B$ is a $K_{\sigma}(X)$, then $A$ is analytic in $X$ iff $A$ is Souslin $K(X)$.

6. Relation between analytic and Borelian sets. Throughout this section we suppose:

(i) $X$ and $X^{\prime}$ are Hausdorff spaces, $X$ is compact.

(ii) For every $C \in K\left(X^{\prime}\right)$ and $C^{\prime} \in K\left(X^{\prime}\right), C-C^{\prime}$ is a $K_{\sigma}\left(X^{\prime}\right)$.

(iii) For every $n \in \omega$ and $k \in \omega, d(n, k)$ is compact in $X^{\prime}$ and for every $N \in \omega$,

$$
D=\bigcap_{n \in \omega} \bigcup_{k \in \omega} d(n, k)=\bigcap_{n \in \omega} \bigcup_{k \in \omega} d(N+n, k) .
$$

(iv) $f$ is a continuous function on $D$ to $X$.

The aim of this section is to prove 6.3 and the much stronger result 6.9. The key points of the proof of 6.9 are contained in 6.2 and 6.7.

6.1. Definitions. Let

$$
\begin{aligned}
& \alpha_{n}(A)=\bigcup_{k^{\prime} \in \omega} \cup\left(f(D \cap A \cap d(n, k)) \cap f\left(D \cap A \cap d\left(n, k^{\prime}\right)-d(n, k)\right)\right), \\
& \alpha_{n}^{\prime}(A)=\bigcup_{i \in(\omega-n)} \alpha_{i}(A) .
\end{aligned}
$$


6.2. Lemma. Let $A$ be analytic in $X^{\prime}, A \subset D, N \in \omega$. If $B$ is analytic in $X$, $B \subset f(A)$ and $B \cap \alpha_{N}^{\prime}(A)=0$ then there exists $B^{\prime}$ such that $B^{\prime}$ is Borelian $K(X)$ and $B \subset B^{\prime} \subset f(D)$.

Proof. Let

$$
E=\{(x, y): y \in A \text { and } x=f(y)\},
$$

$\bar{E}$ be the closure of $E$ in $X \times X^{\prime}$ and, for $n \in \omega$ and $k \in \omega$,

$$
C(n, k)=\bar{E} \cap(X \times d(N+n, k)) .
$$

Then $C(n, k)$ is compact in $X \times X^{\prime}$ and, by Lemma 3.3

$$
\begin{aligned}
f(D) \supset \pi(\bar{E} \cap(X \times D)) & =\pi\left(\bigcap_{n \in \omega} \bigcup_{k \in \omega} C(n, k)\right) \\
& \supset \pi\left(\bigcup_{\bullet \in S^{\prime}} \bigcap_{n \in \omega} C\left(n, s_{n}\right)\right) .
\end{aligned}
$$

Let

$$
d^{\prime}(n, k)=d(n, k)-\bigcup_{\in \in} d(n, j)
$$

and, for every $n \in \omega$ and $p \in S_{n}$,

$$
H(p)=f\left(A \cap \bigcap_{i \in n} d^{\prime}\left(N+i, p_{i}\right)\right) .
$$

Then $H(p)$ is analytic in $X$,

$$
\begin{aligned}
& H(p) \subset \pi\left(\bigcap_{j \in n} C\left(j, p_{j}\right)\right), \\
& \quad B \subset f(A)=f(A \cap D)=\bigcap_{n \in \omega} \bigcup_{p \in S_{n}} B(p) .
\end{aligned}
$$

Moreover, since $B \cap \alpha_{N}^{\prime}(A)=0$, if $p \neq \neq \in \in S_{n}$ we have

$$
B \cap H(p) \cap H(q)=0 .
$$

Then, by 4.3 and 5.5 and induction, for every $n \in \omega$ and $p \in S_{n}$, there exists $\beta(p)$ such that: $\beta(p)$ is Borelian $K(X) ; B \cap H(p) \subset \beta(p)$; if $p \neq q \in S_{n}$ then $\beta(p) \cap \beta(q)=0$;

$$
\beta(p) \subset \beta(p /(n-1)) \text { and } \beta(p) \subset \pi\left(\bigcap_{j \in n} C\left(j, p_{j}\right)\right) .
$$

Let

$$
B^{\prime}=\bigcap_{n \in \omega} \bigcup_{p \in S_{n}} \beta(p)
$$


Then $B^{\prime}$ is Borelian $K(X)$ and

$$
\begin{aligned}
B & =\bigcap_{n \in \omega} \bigcup_{p \in S_{n}}(B \cap H(p)) \subset \bigcap_{n \in \omega} \underset{p \in S_{n}}{\bigcup} \beta(p)=(\text { by 3.2) } \\
& =\bigcup_{s \in S^{\prime}} \bigcap_{n \in \omega} \beta(s / n) \subset \bigcup_{s \in S^{\prime}} \bigcap_{n \in \omega} \pi\left(\bigcap_{j \in n} C\left(j, s_{j}\right)\right)=(\text { by 3.1) } \\
& =\pi\left(\bigcup_{s \in S^{\prime}} \bigcap_{j \in \omega} C\left(j, s_{j}\right)\right) \subset f(D) .
\end{aligned}
$$

Thus, $B \subset B^{\prime} \subset f(D)$.

6.3. TheOREM. Under hypotheses (i) and (ii) at the top of this section, every one-to-one continuous image of a $K_{\sigma \delta}\left(X^{\prime}\right)$ in $X$ is Borelian $K(X)$.

6.4. Lemma. If $A$ and $B$ are Borelian $K\left(X^{\prime}\right)$ then $A-B$ is Borelian $K\left(X^{\prime}\right)$.

Proof. Let $H$ be a maximal family of Borelian $K\left(X^{\prime}\right)$ sets containing $K\left(X^{\prime}\right)$ and such that if $A \in H$ and $B \in H$ then $A-B$ is Borelian $K\left(X^{\prime}\right)$. We easily check that $H$ is closed under countable unions and intersections so that $H$ consists of all Borelian $K\left(X^{\prime}\right)$ sets.

6.5. Lemma. If $A$ is Borelian $K(X)$ then $f^{-1}(A)$ is Borelian $K\left(X^{\prime}\right)$.

Proof. If $A \in K(X)$ then $A$ is closed in $X$ and $f^{-1}(A)$ is closed in $D$ and hence $f^{-1}(A)$ is a $K_{o \delta}\left(X^{\prime}\right)$. Moreover, if, for every $n \in \omega, f^{-1}\left(A_{n}\right)$ is Borelian $K\left(X^{\prime}\right)$ then

and

$$
f^{-1}\left(\bigcup_{n \in \omega} A_{n}\right)=\bigcup_{n \in \omega} f^{-1}\left(A_{n}\right) \text { is Borelian } K\left(X^{\prime}\right)
$$

$$
f^{-1}\left(\bigcap_{n \in \omega} A_{n}\right)=\bigcap_{n \in \omega} f^{-1}\left(A_{n}\right) \text { is Borelian in } K\left(X^{\prime}\right) .
$$

Thus, the set of all $A$ such that $f^{-1}(A)$ is Borelian $K\left(X^{\prime}\right)$ contains $K(X)$ and is closed under countable unions and intersections.

6.6. Definition. Let $F_{n}$ be the set of all finite families $\left\{\Delta_{0}, \cdots, \Delta_{m-1}\right\}$, for any $m \in \omega$, such that for every $i \in m: \Delta_{i} \in K\left(X^{\prime}\right) ; \Delta_{i} \subset d\left(n_{i}, k_{i}\right)$ for some $n \leqq n_{i} \in \omega$ and $k_{i} \in \omega ; \Delta_{i} \cap \Delta_{j}=0$ if $i \neq j \in m$.

6.7. Lemma. Let $0<m \in \omega,\left\{\Delta_{0}, \cdots, \Delta_{m-1}\right\} \in F_{n}$ and $C=\bigcap_{i \in m} f\left(D \cap \Delta_{i}\right)$. If there is no $C^{\prime}$ such that $C^{\prime}$ is Borelian $K(X)$ and $C \subset C^{\prime} \subset f(D)$ then for some $n^{\prime}, n<n^{\prime} \in \omega$ and, for every $i \in m$, there exist $\Delta_{i}^{\prime}$ and $\Delta_{i}^{\prime \prime}$ such that $\Delta_{i}^{\prime} \cup \Delta_{i}^{\prime \prime}$ $\subset \Delta_{i},\left\{\Delta_{0}^{\prime}, \Delta_{0}^{\prime \prime}, \cdots, \Delta_{m-1}^{\prime}, \Delta_{m-1}^{\prime \prime}\right\} \in F_{n^{\prime}}$ and there is no $C^{\prime}$ such that $C^{\prime}$ is Borelian $K(X)$ and

$$
\bigcap_{i \in m}\left(f\left(D \cap \Delta_{i}^{\prime}\right) \cap f\left(D \cap \Delta_{i}^{\prime \prime}\right)\right) \subset C^{\prime} \subset f(D) .
$$


Proof. Suppose the lemma is false. Then, if $n^{\prime} \in S_{m}$ with $n_{i}^{\prime} \geqq n$ for every $i \in m, k \in S_{m}$ and $k^{\prime} \in S_{m}$, there exists $C^{\prime}$ such that $C^{\prime}$ is Borelian $K(X)$ and $\bigcap_{i \in m}\left(f\left(D \cap \Delta_{i} \cap d\left(n_{i}^{\prime}, k_{i}\right)\right) \cap f\left(D \cap \Delta_{i} \cap d\left(n_{i}^{\prime}, k_{i}^{\prime}\right)-d\left(n_{i}^{\prime}, k_{i}\right)\right)\right) \subset C^{\prime} \subset f(D)$.

Hence, recalling definition 6.1 , since $S_{m}$ is countable, there exists $C^{\prime \prime}$ such that $C^{\prime \prime}$ is Borelian $K(X)$ and

$$
\bigcap_{i \in m} \alpha_{n_{i}^{\prime}}\left(\Delta_{i}\right) \subset C^{\prime \prime} \subset f(D) .
$$

Let

$$
B_{0}=\bigcap_{i \in m} \alpha_{n}^{\prime}\left(\Delta_{i}\right)
$$

If $\bar{S}_{m}=\left\{n^{\prime}: n^{\prime} \in S_{m}\right.$ and $\left.n_{i}^{\prime} \geqq n\right\}$, we see that $\bar{S}_{m}$ is countable and

$$
B_{0}=\bigcup_{n^{\prime} \in \bar{S}_{m}} \bigcap_{i \in m} \alpha_{n_{i}^{\prime}}\left(\Delta_{i}\right)
$$

so that there exists $B_{0}^{\prime}$ such that $B_{0}^{\prime}$ is Borelian $K(X)$ and $B_{0} \subset B_{0}^{\prime} \subset f(D)$. Let

$$
D_{0}^{\prime}=f^{-1}\left(B_{0}^{\prime}\right) .
$$

Then, by $6.5, D_{0}^{\prime}$ is Borelian $K\left(X^{\prime}\right)$ and, by $6.4,\left(D \cap \Delta_{i}-D_{0}^{\prime}\right)$ is also Borelian $K\left(X^{\prime}\right)$. Let

$$
C_{0}^{\prime}=\bigcap_{i \in m} f\left(D \cap \Delta_{i}-D_{0}^{\prime}\right) .
$$

Then $C_{0}^{\prime}$ is analytic in $X, C_{0}^{\prime}=C-B_{0}^{\prime}$, and

$$
\bigcap_{i \in m} \alpha_{n}^{\prime}\left(\Delta_{i}-D_{0}^{\prime}\right) \subset \bigcap_{i \in m} \alpha_{n}^{\prime}\left(\Delta_{i}\right)-B_{0}^{\prime}=0 .
$$

For every $j \in m$, let

$$
\beta(j)=C_{0}^{\prime} \cap \bigcap_{i \in m-\{j\}} \alpha_{n}^{\prime}\left(\Delta_{i}-D_{0}^{\prime}\right) .
$$

Then $\beta(j)$ is analytic in $X$ and $\beta(j) \cap \alpha_{n}^{\prime}\left(\Delta_{j}-D_{0}^{\prime}\right)=0$. Hence, by Theorem 6.2 , there exists $\beta^{\prime}(j)$ such that $\beta^{\prime}(j)$ is Borelian $K(X)$ and

$$
\beta(j) \subset \beta^{\prime}(j) \subset f(D) .
$$

Let

$$
\begin{aligned}
& B_{1}^{\prime}=\bigcup_{j \in m} \beta^{\prime}(j), \\
& D_{1}^{\prime}=D_{0}^{\prime} \cup f^{-1}\left(B_{1}^{\prime}\right) \\
& C_{1}^{\prime}=\bigcap_{i \in m} f\left(D \cap \Delta_{i}-D_{1}^{\prime}\right) .
\end{aligned}
$$


Then $B_{1}^{\prime}$ is Borelian $K(X), C_{1}^{\prime}$ is analytic in $X, C_{1}^{\prime}=C-B_{0}^{\prime}-B_{1}^{\prime}$, and for every $j \in m$,

$$
\bigcap_{i \in m-\{j\}} \alpha_{n}^{\prime}\left(\Delta_{i}-D_{1}^{\prime}\right)=0 .
$$

Next, for $j_{1} \in m$ and $j_{2} \in m$, let

$$
\beta\left(j_{1}, j_{2}\right)=C_{1}^{\prime} \cap \bigcap_{i \in m-\left\{j_{1}, j_{2}\right\}} \alpha_{n}^{\prime}\left(\Delta_{i}-D_{1}^{\prime}\right) .
$$

Again, $\beta\left(j_{1}, j_{2}\right)$ is analytic in $X$ and

$$
\beta\left(j_{1}, j_{2}\right) \cap \alpha_{n}^{\prime}\left(\Delta_{j_{1}}-D_{1}^{\prime}\right)=0 .
$$

Hence, by 6.2 , there exists $\beta^{\prime}\left(j_{1}, j_{2}\right)$ such that $\beta^{\prime}\left(j_{1}, j_{2}\right)$ is Borelian $K(X)$ and

$$
\beta\left(j_{1}, j_{2}\right) \subset \beta^{\prime}\left(j_{1}, j_{2}\right) \subset f(D) .
$$

Let

$$
\begin{aligned}
& B_{2}^{\prime}=\bigcup_{j_{1} \in m} \underset{j_{2} \in m}{\bigcup} \beta^{\prime}\left(j_{1}, j_{2}\right), \\
& D_{2}^{\prime}=D_{1}^{\prime} \cup f^{-1}\left(B_{2}^{\prime}\right), \\
& C_{2}^{\prime}=\bigcap_{i \in m} f\left(D \cap \Delta_{i}-D_{2}^{\prime}\right) .
\end{aligned}
$$

Then $B_{2}^{\prime}$ is Borelian $K(X), C_{2}^{\prime}$ is analytic in $X, C_{2}^{\prime}=C-B_{0}^{\prime}-B_{1}^{\prime}-B_{2}^{\prime}$ and for every $j_{1} \in m$ and $j_{2} \in m$,

$$
\bigcap_{i \in m-\left\{j_{1}, j_{2}\right\}} \alpha_{n}^{\prime}\left(\Delta_{i}-D_{2}^{\prime}\right)=0 .
$$

Proceeding this way, we see by induction that, for every $i \in m$, there exists $B_{i}^{\prime}$ such that $B_{i}^{\prime}$ is Borelian $K(X)$ and

$$
C \subset \bigcup_{i \in m} B_{i}^{\prime} \subset f(D)
$$

in contradiction to the hypothesis of the lemma.

6.8. Lemma. If $m \in \omega$, for every $i \in m, C_{i}$ is compact in $X$ and $\bigcap_{i \in m} C_{i}=0$ then, for every $i \in m$, there exists $A_{i}$ such that $A_{i}$ is open, $C_{i} \subset A_{i}$ and

$$
\bigcap_{i \in m} A_{i}=0 .
$$

Proof. We use induction. The lemma is clearly true for $m=2$. Suppose it is true for a given $m \geqq 2$ and let $C_{i}$ be compact in $X$ for every $i \in(m+1)$ and

$$
\bigcap_{i \in(m+1)} C_{i}=0 .
$$




$$
C^{\prime}=C_{m} \cap C_{m-1} \text {. }
$$

Then

$$
C^{\prime} \cap \bigcap_{i \in m-1} C_{i}=0,
$$

so that by the induction hypothesis there exist open sets $A^{\prime}$ and $A_{i}$, for every $i \in m-1$, such that $C^{\prime} \subset A^{\prime}, C_{i} \subset A_{i}$ and

$$
A^{\prime} \cap \bigcap_{i \in m-1} A_{i}=0 .
$$

Since $\left(C_{m}-A^{\prime}\right) \cap\left(C_{m-1}-A^{\prime}\right)=0$, let $A_{m}^{\prime}$ and $A_{m-1}^{\prime}$ be open,

$$
C_{m}-A^{\prime} \subset A_{m}^{\prime}, \quad C_{m-1}-A^{\prime} \subset A_{m-1}^{\prime}, \quad A_{m}^{\prime} \cap A_{m-1}^{\prime}=0 .
$$

Let

$$
A_{m}=A^{\prime} \cup A_{m}^{\prime} \text { and } A_{m-1}=A^{\prime} \cup A_{m-1}^{\prime} .
$$

Then, for every $i \in(m+1), A_{i}$ is open, $C_{i} \subset A_{i}$ and

$$
\bigcap_{i \in(m+1)} A_{i}=\left(A^{\prime} \cup A_{m}^{\prime}\right) \cap\left(A^{\prime} \cup A_{m-1}^{\prime}\right) \cap \bigcap_{i \in m-1} A_{i}=A^{\prime} \cap \bigcap_{i \in m-1} A_{i}=0 .
$$

6.9. THEOREM. Under hypotheses (i) and (ii) at the top of this section, every countable-to-one continuous image of a $K_{\sigma \delta}\left(X^{\prime}\right)$ in $X$ is Borelian $K(X)$.

Proof. Suppose the theorem is false. Let

$$
\begin{aligned}
& S_{n}=\left\{p: p \in S_{n} \text { and, for every } i \in n, p_{i}=0 \text { or } p_{i}=1\right\}, \\
& s^{\prime}=\left\{s: s \in S^{\prime} \text { and, for every } i \in \omega, s_{i}=0 \text { or } s_{i}=1\right\} .
\end{aligned}
$$

In view of Lemma 6.7, using induction we see that for every $n \in \omega$ and $p \in S_{n}$ there exist $N_{n} \in \omega$ and $\Delta(p)$ such that: $N_{n}<N_{n+1}, \Delta(p) \subset \Delta(p /(n-1))$ for $n>0$,

$$
\bigcup_{p \in \mathcal{S}_{n}}\{\Delta(p)\} \in F_{N_{n}},
$$

and there is no $C_{n}$ such that $C_{n}$ is Borelian $K(X)$ and

$$
\bigcap_{p \in \mathcal{S}_{n}} f(D \cap \Delta(p)) \subset C_{n} \subset f(D) .
$$

In particular,

$$
\bigcap_{p \in \mathcal{S}_{n}} f(D \cap \Delta(p)) \neq 0 .
$$

For every $s \in \mathcal{S}^{\prime}$, let 


$$
\bar{\Delta}(s)=\bigcap_{n \in \omega} \Delta(s / n)
$$

so that $\bar{\Delta}(s)$ is compact in $X^{\prime}$ and $\bar{\Delta}(s) \subset D$.

We now check the following

Statement. If $0<m \in \omega$ and, for every $i \in m, s^{(i)} \in \mathcal{S}^{\prime}$ then

$$
\bigcap_{i \in m} f\left(\bar{\Delta}\left(s^{(i)}\right)\right) \neq 0 .
$$

If the statement is false, since $f\left(\bar{\Delta}\left(s^{(i)}\right)\right)$ is compact in $X$, by Lemma 6.7, there exist open sets $A_{i}$ such that $f\left(\bar{\Delta}\left(s^{(i)}\right)\right) \subset A_{i}$ and $\bigcap_{i \in m} A_{i}=0$. Let $A_{i}^{\prime}$ $=f^{-1}\left(A_{i}\right)$. Then for some $n^{\prime} \in \omega$ we have, for every $i \in m$,

$$
D \cap \Delta\left(s^{(i)} / n^{\prime}\right) \subset A_{i}^{\prime},
$$

hence

$$
f\left(D \cap \Delta\left(s^{(i)} / n^{\prime}\right)\right) \subset A_{i}
$$

and

$$
\bigcap_{p \in \mathcal{S}_{n^{\prime}}} f(D \cap \Delta(p)) \subset \bigcap_{i \in m} f\left(D \cap \Delta\left(s^{(i)} / n^{\prime}\right)\right) \subset \bigcap_{i \in m} A_{i}=0
$$

in contradiction to (I) above.

Returning to the proof of the theorem, we conclude from the above Statement that

$$
\bigcap_{s \in \mathcal{S}^{\prime}} f(\bar{\Delta}(s)) \neq 0 .
$$

Moreover, if $s \in \mathcal{S}^{\prime}, t \in \mathcal{S}^{\prime}$ and $s \neq t$ then $\bar{\Delta}(s) \cap \bar{\Delta}(t)=0$. Thus, if

$$
y \in \bigcap_{s \in \mathcal{S}^{\prime}} f(\bar{\Delta}(s)) \text {, }
$$

since $\mathcal{S}^{\prime}$ is noncountable, we conclude that $D \cap f^{-1}\{y\}$ is noncountable, in contradiction to the hypothesis.

6.10. Corollary. Suppose $X^{\prime}$ is Hausdorff and, for every $C \in K\left(X^{\prime}\right)$ and $C^{\prime} \in K\left(X^{\prime}\right), C-C^{\prime}$ is a $K_{\sigma}\left(X^{\prime}\right) ; D$ is a $K_{\sigma \delta}\left(X^{\prime}\right) ; Y$ is Hausdorff and the countable union of compact sets. If $f$ is continuous on $D$ to $Y$ and, for every $y, f^{-1}\{y\}$ is countable then $f(D)$ is Borelian $K(Y)$.

\section{BIBLIOGRAPHY}

1. G. Choquet, Ensembles boréliens et analytiques dans les espaces topologiques, C. R. Acad. Sci. Paris, vol. 232 (1951) pp. 2174-2176. 295.

2. - Theory of capacities, Ann. Inst. Fourier, Grenoble, vol. 5 (1953-1954) pp. 131-

3. - Ensembles $K$-analytiques et $K$-sousliniens. Cas général et cas métrique, Ann. Inst. Fourier vol. 9 (1959) pp. 75-81. 
4. F. Hausdorff, Mengenlehre, 3rd ed., Berlin, 1935.

5. N. Luzin, Leçons sur les ensembles analytiques et leurs applications, Paris, 1930.

6. S. Saks, Theory of the integral, Warsaw, 1937, p. 50.

7. W. Sierpiński, Les ensembles projectifs et analytiques, Paris, 1950.

8. V. E. Sneider, Continuous images of Suslin and Borel sets. Metrization theorems (in Russian), Dokl. Akad. Nauk SSSR (N.S.) vol. 50 (1945) pp. 77-79.

9. - Descriptive theory of sets in topological spaces (in Russian), Dokl. Akad. Nauk SSSR (N.S.) vol. 50 (1945) pp. 81-83.

10. - Descriptive theory of sets in topological spaces (in Russian), Uĉ. Zap. Moskov. Gos. Univ. vol. 135, tom II (1948) pp. 37-85.

University of California,

Berkeley, California 